\title{
A COEFFICIENT INEQUALITY FOR CERTAIN CLASSES OF ANALYTIC FUNCTIONS
}

\author{
F. R. KEOGH ${ }^{1}$ AND E. P. MERKES
}

1. Statement of results. If $f(z)=z+\sum_{n=2}^{\infty} a_{n} z^{n}$ is analytic and univalent in the unit disk $E(|z|<1)$, then it is known [1] that

$$
\begin{aligned}
\left|a_{3}-\mu a_{2}^{2}\right| & \leqq 4 \mu-3 \text { when } \mu \geqq 1, \\
& \leqq 1+2 \exp [-2 \mu /(1-\mu)] \quad \text { when } 0 \leqq \mu \leqq 1, \\
& \leqq 3-4 \mu \text { when } \mu \leqq 0 .
\end{aligned}
$$

The result is sharp in the sense that for each $\mu$ there is a function in the class under consideration for which equality holds.

This paper contains analogues of (1) for certain classes of analytic functions. Explicitly, let $\gamma$ and $\lambda$ be real numbers, where $|\gamma|<\pi / 2$ and $0 \leqq \lambda<1$, and let $S(\gamma, \lambda)$ denote the class of analytic functions $f(z)^{\prime}$ in $E$ such that $f(0)=0, f^{\prime}(0)=1$ and

$$
\operatorname{Re}\left\{e^{i \gamma} \frac{z f^{\prime}(z)}{f(z)}\right\}>\lambda \cos \gamma \quad(z \in E) .
$$

In particular, $S(0, \lambda)$ is Robertson's class of functions that are starlike of order $\lambda$ in $E[6]$ and $S(0,0)$ is the class of normalized starlike functions. The following sharp result is proved in $\$ 2$.

THEOREM 1. If $f(z)=z+\sum_{n=2}^{\infty} a_{n} z^{n}$ is in $S(\gamma, \lambda)$ and if $\mu$ is a complex number, then

(3) $\left|a_{3}-\mu a_{2}^{2}\right| \leqq(1-\lambda) \cos \gamma \max \left(1,\left|2 \cos \gamma(1-\lambda)(2 \mu-1)-e^{i \gamma}\right|\right)$.

For each $\mu$, there is a function in $S(\gamma, \lambda)$ for which equality holds.

Hummel ([2], [3]), using variational techniques, proves the conjecture of V. Singh that $\left|a_{3}-a_{2}^{2}\right| \leqq 1 / 3$ for the normalized convex functions in $E$. Since $z f^{\prime}(z)$ is starlike if and only if $f(z)$ is convex in $E[5$, p. 223], the following extension of this result is obtained.

CoROLlaRY 1. If $f(z)=z+\sum_{n=2}^{\infty} a_{n} z^{n}$ is analytic and convex in $E$ and if $\mu$ is a complex number, then $\left|a_{3}-\mu a_{2}^{2}\right| \leqq \max (1 / 3,|\mu-1|)$. The result is sharp for each $\mu$.

Presented to the Society, January 24, 1968; received by the editors July 15, 1967 .and, in revised form, October 18, 1967.

${ }^{1}$ Supported by the National Science Foundation Grant GP 7377. 
A function $f(z)$ is spiral-like [7] in $E$ if there is a real $\gamma,|\gamma|<\pi / 2$, such that $f(z) \in S(\gamma, 0)$. Another simple consequence of Theorem 1 is

CoROLlaRY 2. If $f(z)=z+\sum_{n=2}^{\infty} a_{n} z^{n}$ is spiral-like in $E$ and if $\mu$ is a complex number, then

$$
\left|a_{3}-\mu a_{2}^{2}\right| \leqq 2|\mu-1|+|2 \mu-1| .
$$

For each real $\mu$, there is a starlike function for which equality holds.

An analytic function $f(z)=z+\cdots$ in $E$ is close-to-convex [4] if there is a real $\gamma,|\gamma|<\pi / 2$, and a starlike function $g(z)=z+\cdots$ such that

$$
\operatorname{Re}\left\{e^{i \gamma} \frac{z f^{\prime}(z)}{g(z)}\right\}>0 \quad(z \in E) .
$$

In $\S 3$ we prove

THEOREM 2. If the analytic function $f(z)=z+\sum_{n=2}^{\infty} a_{n} z^{n}$ in $E$ is close-to-convex and if $\mu$ is a real number, then

$$
\left|a_{3}-\mu a_{2}^{2}\right| \leqq \max (1,3|\mu-1|,|4 \mu-3|) .
$$

If $\mu$ is outside the interval $(0,2 / 3)$, there is an analytic close-toconvex function for which equality holds.

Let $K_{0}$ be the subclass of analytic close-to-convex functions $f(z)$ such that (4) holds with $\gamma=0$ for some starlike function $g(z)$ $=z+\cdots$ in $E$. In $\$ 4$ we prove the following sharp result.

TheOREM 3. If $f(z)=z+\sum_{n=2}^{\infty} a_{n} z^{n}$ is in $K_{0}$ and if $\mu$ is real, then

$$
\begin{aligned}
\left|a_{3}-\mu a_{2}^{2}\right| & \leqq 3-4 \mu \text { for } \mu \leqq 1 / 3, \\
& \leqq 1 / 3-4 / 9 \mu \text { for } 1 / 3 \leqq \mu \leqq 2 / 3, \\
& \leqq 1 \text { for } 2 / 3 \leqq \mu \leqq 1 \\
& \leqq 4 \mu-3 \text { for } \mu \geqq 1
\end{aligned}
$$

For each $\mu$, there is a function in $K_{0}$ such that equality holds.

We suspect that the bounds in (6) are sharp when $\mu \in(0,2 / 3)$ for the wider class of all analytic close-to-convex functions.

2. Proof of Theorem 1. First, if $\Phi(z)=\sum_{n=1}^{\infty} \alpha_{n} z^{n}$ is in the class $B$ of functions that are analytic in $E$ and map the unit disk into itself, then $\left|\alpha_{2}\right| \leqq 1-\left|\alpha_{1}^{2}\right|$ (for example, see [5, p. 108]). Therefore, if $s$ is a complex number, we have 


$$
\begin{aligned}
\left|\alpha_{2}-s \alpha_{1}^{2}\right| & \leqq\left|\alpha_{2}\right|+|s|\left|\alpha_{1}^{2}\right| \leqq 1+(|s|-1)\left|\alpha_{1}^{2}\right| \\
& \leqq \max (1,|s|) .
\end{aligned}
$$

Moreover, the functions $\Phi(z)=z$ and $\Phi(z)=z^{2}$ respectively show that the result is sharp for $|s| \geqq 1$ and for $|s|<1$. Now, by (2), $f(z)$ $=z+\sum_{n=2}^{\infty} a_{n} z^{n}$ is in $S(\alpha, \lambda)$ if and only if the function

$$
\Phi(z)=\frac{f^{\prime}(z)-f(z) / z}{f^{\prime}(z)+\left[(1-\lambda) e^{-2 i \gamma}-\lambda\right] f(z) / z}=\sum_{n=1}^{\infty} \alpha_{n} z^{n}
$$

is in the class $B$. A simple computation shows

(8) $\quad \alpha_{1}=\frac{u a_{2}}{1-\lambda}, \quad \alpha_{2}=\frac{2 u}{1-\lambda}\left[a_{3}-\frac{1-\lambda-u}{2(1-\lambda)} a_{2}^{2}\right], \quad u=\frac{e^{i \gamma}}{2 \cos \gamma}$.

The inequality (3) with

$$
\mu=\frac{1-\lambda+(s+1) u}{2(1-\lambda)}
$$

is now obtained by substituting the coefficients (8) into (7). That (3) is sharp follows from the sharpness of the inequalities (7).

REMARK. The same argument also proves

$$
\begin{aligned}
\left|a_{3}-\mu a_{2}^{2}\right| \leqq & (1-\lambda) \cos \gamma+\left(\left|2 \cos \gamma(1-\lambda)(2 \mu-1)-e^{i \gamma}\right|-1\right) \\
& \cdot\left|a_{2}^{2}\right| / 4(1-\lambda) \cos \gamma .
\end{aligned}
$$

For each $a_{2}$, where $\left|a_{2}\right|<2(1-\lambda) \cos \gamma$, and for each complex number $\mu$, there is a function in $S(\gamma, \lambda)$ for which equality holds.

3. Proof of Theorem 2. By (4) the analytic function $f(z)$ $=z+\sum_{n=2}^{\infty} a_{n} z^{n}$ in $E$ is close-to-convex if and only if there exists a $g(z)=z+\sum_{n=2}^{\infty} c_{n} z^{n}$ in $S(0,0)$ such that the function

$$
\Phi(z)=e^{i \gamma} \frac{f^{\prime}(z)-g(z) / z}{f^{\prime}(z)+e^{-2 i \gamma} g(z) / z}=\sum_{n=1}^{\infty} \alpha_{n} 2^{n}
$$

is in the class $B$ of $\$ 2$. A comparison of the coefficients in the various power series expansions for the functions in this identity shows

$$
2 a_{2}=c_{2}+2 \cos \gamma \alpha_{1}, \quad 3 a_{3}=c_{3}+2 \cos \gamma\left(\alpha_{1} c_{2}+\alpha_{2}+e^{i \gamma} \alpha_{1}^{2}\right) .
$$

Therefore, we have

$$
\begin{aligned}
a_{3}-\mu a_{2}^{2}= & \frac{1}{3}\left(c_{3}-\frac{3}{4} \mu c_{2}^{2}\right)+\frac{2}{3} \cos \gamma\left[\alpha_{2}+\left(e^{i \gamma}-\frac{3}{2} \mu \cos \gamma\right) \alpha_{1}^{2}\right] \\
& +\left(\mu-\frac{2}{3}\right) \cos \gamma \alpha_{1} c_{2} .
\end{aligned}
$$


Set $\mu=2 / 3$. By (7) and Theorem 1 , we obtain

$$
\begin{aligned}
\left|a_{3}-\frac{2}{3} a_{2}^{2}\right| & \leqq \frac{1}{3}\left|c_{3}-\frac{1}{2} c_{2}^{2}\right|+\frac{2}{3} \cos \gamma\left|\alpha_{2}+i \sin \gamma \alpha_{1}^{2}\right| \\
& \leqq \frac{1}{3}+\frac{2}{3} \cos \gamma \leqq 1
\end{aligned}
$$

From the Area Theorem [5, p. 210], we have $\left|a_{3}-a_{2}^{2}\right| \leqq 1$ and by (9), we get $\left|a_{3}\right| \leqq 3$. Thus for $0 \leqq \mu \leqq 2 / 3$, it follows that

$$
\left|a_{3}-\mu a_{2}^{2}\right| \leqq \frac{3}{2} \mu\left|a_{3}-\frac{2}{3} a_{2}^{2}\right|+\left(1-\frac{3}{2} \mu\right)\left|a_{3}\right| \leqq 3(1-\mu)
$$

and, for $2 / 3 \leqq \mu \leqq 1$, that

$$
\left|a_{3}-\mu a_{2}^{2}\right| \leqq(3 \mu-2)\left|a_{3}-a_{2}^{2}\right|+3(1-\mu)\left|a_{3}-2 a_{2}^{2} / 3\right| \leqq 1 .
$$

The last result is sharp since the close-to-convex class include the starlike functions $S(0,0)$ and the inequality is sharp in the latter class by Theorem 1 . Finally, if $\mu$ is not in the interval $[0,1]$, then by (1) $\left|a_{3}-\mu a_{2}^{2}\right| \leqq|4 \mu-3|$ since the close-to-convex functions are univalent $[4]$.

4. Proof of Theorem 3. From (7), (9) with $\gamma=0$ and Theorem 1 for the starlike class, we have

$$
\begin{aligned}
\left|a_{3}-\mu a_{2}^{2}\right| \leqq & \frac{1}{3}\left\{1+\frac{1}{4}[|3 \mu-3|-1]\left|c_{2}^{2}\right|\right\} \\
& +\frac{2}{3}\left\{1+\frac{1}{2}[|3 \mu-2|-2]\left|\alpha_{1}^{2}\right|\right\} \\
& +\frac{1}{3}|3 \mu-2|\left|\alpha_{1}\right|\left|c_{2}\right| .
\end{aligned}
$$

If $1 / 3 \leqq \mu \leqq 2 / 3$, this becomes

$$
\begin{aligned}
\left|a_{3}-\mu a_{2}^{2}\right| \leqq & +\frac{1}{12}\left\{(2-3 \mu)\left|c_{2}^{2}\right|\right. \\
& \left.+4(2-3 \mu)\left|\alpha_{1}\right|\left|c_{2}\right|-12 \mu\left|\alpha_{1}^{2}\right|\right\} \\
= & 1+\frac{1}{12}\left\{2-3 \mu+\frac{(2-3 \mu)^{2}}{3 \mu}\right\}\left|c_{2}^{2}\right| \\
& -\mu\left\{\left|\alpha_{1}\right|-\frac{(2-3 \mu)}{6 \mu}\left|c_{2}\right|\right\}^{2} \\
\leqq & 1+\frac{2-3 \mu}{18 \mu}\left|c_{2}^{2}\right| \leqq \frac{1}{3}+\frac{4}{9 \mu},
\end{aligned}
$$

since $\left|c_{2}\right| \leqq 2$. The result is sharp since there is a starlike function (the Koebe function $g(z)=z /(1-z)^{2}$ ) with $c_{2}=2, c_{3}=3$ and a function in $B$ with $\alpha_{1}=(2-3 \mu) / 3 \mu, \alpha_{2}=1-\alpha_{1}^{2}$, provided $1 / 3 \leqq \mu \leqq 2 / 3$. For $0 \leqq \mu \leqq 1 / 3$, we have 


$$
\left|a_{3}-\mu a_{2}^{2}\right| \leqq 3 \mu\left|a_{3}-a_{2}^{2} / 3\right|+(1-3 \mu)\left|a_{3}\right| \leqq 3-4 \mu .
$$

For the remaining choices of $\mu,(6)$ is a consequence of Theorem 2. The sharpness for $\mu$ not in the interval $(1 / 3,2 / 3)$ follows from Theorem 1 , since $S(0,0) \subset K_{0}$.

\section{REFERENCES}

1. M. Fekete and G. Szegö, Eine Bemerkung über ungerade schlichte Funktionen, J. London Math. Soc. 8 (1933), 85-89.

2. J. Hummel, The coefficient regions of starlike functions, Pacific J. Math. 7 (1957), 1381-1389.

3. - Extremal problems in the class of starlike functions, Proc. Amer. Math. Soc. 11 (1960), 741-749.

4. W. Kaplan, Close-to-convex schlicht functions, Michigan Math. J. 1 (1952), 169-185.

5. Z. Nehari, Conformal mapping, McGraw-Hill, New York, 1952.

6. M. S. Robertson, On the theory of univalent functions, Ann. of Math. 37 (1936), 374-408.

7. L. Špaček, Contribution à la théorie des functions univalents, Časopis Pěst. Mat. 62 (1932), 12-19.

UNIVERSITY OF KENTUCKY AND UNIVERSITY OF CinCINNATI 